\title{
Cardiac magnetic resonance imaging (cMRI) as a first-line approach to subclinical heart failure in children undergoing cardiotoxic chemotherapy
}

\author{
Maëlle de Ville de Goyet ${ }^{*}$, Benedicte Brichard, Thierry Pirotte, Stephane Moniotte \\ From 2011 SCMR/Euro CMR Joint Scientific Sessions \\ Nice, France. 3-6 February 2011
}

\section{Background}

Cardiotoxic chemotherapy including anthracyclines remains a standard of care in paediatric cancers. Recent data suggest that heart failure secondary to chemotherapy is, to some degree, reversible, but requires early detection and prompt intervention. Since a substantial proportion of paediatric patients still develop heart failure, this work aims to develop a follow-up protocol based on cMRI to evaluate the patient's cardiovascular function and haemodynamics, as well as myocardial iron overload, perfusion and viability.

\section{Material and methods}

From October 2009 to September 2010, 38 children (mean age 7,8 years $(0,3-17,6)$; mean BSA of $0,98 \mathrm{~m}^{2}$ ) were prospectively identified as candidates for cardiotoxic chemotherapy. All patients underwent a cMRI to evaluate 1) systolic and diastolic function on steadystate free precession cine and tagged imaging, 2) myocardial perfusion and viability after Gadolinium injections, 3) mitral and aortic flows through velocityencoded sequences, and 4) myocardial and liver iron content based on T2* MRI.

\section{Results}

The study protocol was completed in all patients within the first month of diagnosis, under general anaesthesia for the 14 youngest patients. The mean MRI acquisition time $40+/-9 \mathrm{~min}$. Systolic biventricular volumes, mass and function as well as left ventricular (LV) diastolic function were normal in all patients, with a mean LV EF of $62,2+/-8 \%$ and RV EF of $49,5+/-7,2 \%$. Although a perfusion defect was not retrieved in any of these prechemotherapy assessments, mild LV myocardial scaring (mean of 4,5\%) was frequently identified by gadoliniumenhanced imaging. Aortic and mitral flux showed normal LV stroke volumes (mean of $3,78 \mathrm{~L} / \mathrm{min} / \mathrm{m}^{2}$ ). No significant liver or cardiac iron overload was detected (mean T2* of 23,6 and 33,2msec, respectively). Evaluation of the inter-observer and intra-observer variability was performed for all variables studied and confirmed that the coefficients of determination were very strong $\left(R^{2}>0,95\right)$, as previously reported by others.

\section{Conclusions}

Our preliminary study shows that a detailed cardiovascular evaluation of young patients undergoing chemotherapy is achievable in a reasonable time in the early phase following diagnosis. We believe that the low intra and inter-observer variability of cMRI makes it suitable for a complete mid- and/or long-term follow-up of cardiovascular function. This should allow to evaluate the true harmful effects of cardiotoxic medications used against paediatric cancers and to confirm the potential role of cMRI as a first-line approach to subclinical heart failure in children.

Published: 2 February 2011

doi:10.1186/1532-429X-13-S1-P177

Cite this article as: de Ville de Goyet et al:: Cardiac magnetic resonance imaging (cMRI) as a first-line approach to subclinical heart failure in children undergoing cardiotoxic chemotherapy. Journal of Cardiovascular Magnetic Resonance 2011 13(Suppl 1):P177. 